\title{
Urbanisation et transition de la fécondité en Albanie
}

\author{
MATHIAS LERCH ${ }^{1}$
}

\begin{abstract}
Résumé
Parallèlement au rapide développement du processus d'urbanisation, la fécondité albanaise a diminué de moitié depuis la fin du régime communiste. Pour vérifier cette apparente corrélation au niveau macro-démographique, une analyse de l'impact de l'exode rural sur les comportements nuptiaux et féconds à l'aide de données individuelles et longitudinales des deux dernières décennies a été effectuée. Resituer ces dynamiques dans leur contexte évolutif s'avère en effet crucial pour la compréhension du rôle de l'exode rural dans la transition de la fécondité albanaise. Nos analyses multivariées comparent le mariage et la fécondité des nonmigrantes à celle des migrantes ainsi que de leurs enfants au cours de leur parcours migratoire. Les résultats montrent que l'exode rural vers les centres urbains n'a pas altéré les comportements familiaux traditionnels en Albanie, mais au contraire y est resté étroitement associé. Ainsi, la transition de la fécondité a été freinée en raison d'une résistance des migrantes face aux transformations que connaît la famille albanaise contemporaine.
\end{abstract}

\section{Mots-clés}

Urbanisation, migrations internes, fécondité, Albanie

\section{Summary}

While Albania's population urbanized at a fast pace, its level of fertility fell by half since the end of communist rule. This apparent correlation at the macro-demographic level is examined through an analysis of the impact of rural exodus on marriage and fertility behaviours. Using individual and longitudinal data for the last twenty years, we situate these dynamics in their changing context. The revelations are indeed crucial in understanding the effect of rural exodus on Albania's fertility transition. Our multivariate analyses compare marriage and fertility patterns of non-migrants to family behaviours among migrants (as well as their descents) during their residential trajectory. Results show that rural outmigration did not alter traditional family behaviours in Albania, which remained closely related to each other. The fertility transition was delayed because of the migrants' resistance to the recent transformations of the contemporary Albanian family.

1. Université de Genève, Suisse. 


\author{
Keywords \\ Urbanisation, internal migration, fertility, Albania
}

\title{
Introduction ${ }^{2}$
}

En 1989, l'Albanie affichait le plus haut niveau de fécondité d'Europe et comptait, avec un tiers de sa population habitant dans les villes, parmi les pays les moins urbanisés. Depuis, la société a connu des transformations économiques et sociales majeures, passant d'une isolation complète sous un régime communiste totalitaire à une soudaine extraversion, une libéralisation économique et un pluralisme politique. Durant les deux dernières décennies, une large part de la population a migré à l'étranger et plus de la moitié des Albanais vit aujourd'hui dans les villes. Parallèlement à cette rapide urbanisation, la fécondité a diminué de moitié pour atteindre 1,6 enfants par femme. Ainsi l'exemple de l'Albanie semble confirmer l'hypothèse de la transition de la mobilité, qui stipule des interrelations entre la baisse de la fécondité et l'augmentation de la mobilité géographique et du niveau d'urbanisation des populations (Zelsinksi, 1971). L'apparente corrélation au niveau macro-démographique entre ces phénomènes est vérifiée ici par une analyse de l'impact de l'exode rural sur les comportements nuptiaux et féconds des femmes albanaises.

L'effet négatif de l'urbanisation sur la fécondité mesurée au niveau national est généralement attribué à la transformation des comportements reproductifs des migrants suite à leur changement de lieu de résidence. Socialisées dans des régions caractérisées par une fécondité élevée, l'évènement migratoire provoque une rupture momentanée du processus de constitution de la famille et les comportements féconds des migrants tendent à converger vers des standards plus faibles prévalant en ville (Goldstein, Goldstein, 1981 ; Brockerhoff, 1998 ; White et al., 1995). Étant donné l'accroissement de la part des femmes habitants les villes au cours de l'urbanisation, la plus faible fécondité urbaine détermine progressivement la transition de la fécondité au niveau national. Ce schéma théorique se réfère au processus universel de modernisation et

2. L'auteur remercie Michel Oris et deux relecteurs anonymes pour leurs remarques constructives qui ont permis d'améliorer la version finale du manuscrit. Merci également à l'Institut Statistique Albanais pour la mise à disposition les données du DHS 2008/9 et du recensement 2001 ainsi qu'à Alain Jarne pour la typologie territoriale utilisée dans le tableau 1. 
fait abstraction du contexte dans lequel les interactions entre migration et fécondité ont lieu. Or, le contexte socioéconomique et culturel influence ces interactions et reste essentiel à leur compréhension (Goldstein, Goldstein, 1983). Resituer ces dynamiques dans le cadre des transformations de l'Albanie depuis 1989 s'avère en effet crucial pour la compréhension du rôle de l'exode rural dans la transition de la fécondité.

La section suivante introduit brièvement le contexte albanais. Les hypothèses concernant l'effet de l'exode rural sur la fécondité des migrantes, et par conséquent sur la transition de la fécondité nationale depuis 1989, sont ensuite spécifiées pour les deux dernières décennies. Suite à la description des données et méthodes, les parcours familiaux et migratoires des femmes sont analysés dans une perspective longitudinale et multivariée. Nous comparons l'entrée en vie reproductive et l'élargissement des familles des non-migrantes aux comportements des migrantes ainsi que de leurs enfants au cours de leur parcours migratoire. Notre analyse teste la sélection des migrantes selon le projet fécond, les effets de rupture de la migration sur le parcours reproductif ainsi que ses interdépendances avec la mobilité géographique. La convergence des comportements des migrantes vers les standards urbains est également évaluée. Les résultats sont enfin discutés au regard du contexte spécifique de l'Albanie.

\section{Contexte albanais}

L'Albanie est un petit pays montagneux situé sur la côte Est de la Mer Adriatique. Il se caractérisait par une démographie atypique par rapport aux autres pays du continent européen. La transition de la fécondité ne s'est amorcée que depuis 1965 - soit avec un décalage d'au moins 15 ans par rapport aux autres pays de la région (Sardon, 2000). Bien que la baisse du nombre d'enfants par femme ait été rapide - de 6 à 3 en 1990 ; la fécondité est restée la plus élevée d'Europe (Falkingham, Gjonca, 2001). Le niveau d'urbanisation de la population est resté faible jusqu'en en 1989, le processus ayant été freiné sous le communisme durant plusieurs décennies.

À la fin de la Seconde Guerre mondiale, moins d'un cinquième des 1,1 millions résidents albanais habitait les villes. Dans les années 1950, la première phase d'industrialisation socialiste a provoqué un intense exode rural. Le gouvernement a ensuite strictement contrôlé et limité la mobilité interne à partir de 1965 (l'émigration internationale était con- 
sidérée comme une trahison d'État et punie de la peine de mort). Diverses mesures visaient à stabiliser la part de la population urbaine, telles que l'introduction de passeports domestiques et permis de résidence, l'allocation régionale de la main-d'œuvre par le gouvernement central ainsi qu'une politique active de rétention des populations dans les zones rurales. Cette politique avait pour but d'augmenter la faible productivité du secteur agricole dans le contexte d'une isolation croissante de l'Albanie sur la scène internationale nécessitant une autosubsistance alimentaire. L'instauration des stricts contrôles de la migration interne a coïncidé avec la révolution culturelle et la fin de la collectivisation des terres agricoles (Borchert, 1975). Par conséquent, le niveau d'urbanisation n'a guère augmenté entre 1960 et 1989 (de $31 \%$ à 36 \% ; Sjöberg, 1994 ; Sjöberg, 1992).

Lors de la chute du régime communiste en 1991, l'Albanie comptait parmi les pays les plus pauvres d'Europe. Selon le recensement de 1989, la majorité de la population travaillait sur les champs agricoles et les logements urbains étaient faiblement équipés d'infrastructures sanitaires de base (Central Directory of Statistics, 1991). Le maintien d'un niveau de fécondité élevé a accentué la pression démographique sur l'économie en crise. La distribution par habitant des terres agricoles, lors de leurs privatisations, n'a pas soulagé cette situation puisqu'elle a entrainé une atomisation des exploitations ne permettant que difficilement l'autosubsistance des ménages (UNDP, 2000). Ainsi, les migrations internes et internationales ont constitué des réponses majeures de la population conséquemment à la nouvelle liberté acquise dans un contexte incertain : jusqu'en 2001, un cinquième de la population a quitté le pays et un quart vivait dans une autre commune que celle douze ans auparavant (INSTAT, 2004). Les flux migratoires ont été les plus importants lors des deux crises majeures : la crise économique et institutionnelle consécutive à l'effondrement du système communiste et la crise financière de 1997, qui ont accentué la pauvreté dans le pays (World Bank, 2007). En 2002, près d'un tiers des résidents des campagnes vivait en dessous du seuil de pauvreté et cela en dépit d'importants flux de transferts de fonds des migrants établis à l'étranger (World Bank, 2003).

Selon nos estimations basées sur le recensement de 2001 et la typologie territoriale proposée par Schuler et al. (2010), depuis 1989, deux tiers des migrants internes provenaient des régions rurales, particulièrement celles situées dans les montagnes (Tableau 1). La majorité de ces migrants s'est établie dans une des villes et plus d'un cinquième dans les communes environnantes, intégrées au tissu urbain. L'exode rural s'est principalement concentré sur la capitale du pays, Tirana, qui totalise 
$14 \%$ des résidents en Albanie. Par conséquent, le niveau officiel d'urbanisation a augmenté de 36 à $42 \%$ entre 1989 et 2001 . Si les frontières des villes avaient été mises à jour, la population urbaine aurait représenté moins de deux tiers des Albanais au recensement de 2001 (Schuler et al., 2010).

TABLEAu 1 Distribution relative des migrants intercommunaux depuis 1989 et des non-migrants selon la typologie urbaine de Schuler (2010), population albanaise âgé de 15 ans et plus, 2001

\begin{tabular}{|c|c|c|c|}
\hline & \multirow{2}{*}{$\begin{array}{l}\text { Non mi- } \\
\text { grants }\end{array}$} & \multicolumn{2}{|c|}{ Migrants } \\
\hline & & Origine & Destination \\
\hline \multicolumn{4}{|l|}{ Agglomérations urbaines } \\
\hline Villes & 37,5 & 19,2 & 50,8 \\
\hline $\begin{array}{l}\text { Communes rurales urbanisées et intégrées mor- } \\
\text { phologiquement aux villes }\end{array}$ & 13,4 & 10,7 & 22,7 \\
\hline \multicolumn{4}{|l|}{ Régions rurales } \\
\hline $\begin{array}{l}\text { Communes industrielles, minières, de services, } \\
\text { touristiques }\end{array}$ & 5,6 & 7,0 & 3,4 \\
\hline Communes majoritairement agricoles des plaines & 8,1 & 7,6 & 5,5 \\
\hline $\begin{array}{l}\text { Communes majoritairement agricoles des mon- } \\
\text { tagnes }\end{array}$ & 9,6 & 19,1 & 3,6 \\
\hline Communes totalement agricoles des plaines & 16,5 & 14,8 & 10,8 \\
\hline Communes totalement agricoles des montagnes & 9,4 & 21,6 & 3,1 \\
\hline Total & 100,0 & 100,0 & 100,0 \\
\hline Effectifs totaux & 1697631 & 412544 & 412544 \\
\hline
\end{tabular}

En comparaison avec la première décennie caractérisée par des crises, la seconde se distingue par une stabilité politique et une modernisation sociale, soutenue par une forte croissance économique et une émigration continue. En dépit de l'importante baisse et convergence des niveaux de pauvreté urbains et ruraux (INSTAT et al., 2009), l'urbanisation rapide poursuit son cours et dépasse la barre des $50 \%$ en 2009, comme l'atteste le niveau officiel.

Parallèlement à ce rattrapage du processus d'urbanisation, l'indice synthétique de fécondité (ISF) a diminué presque de moitié depuis 1989 pour atteindre 1,6 enfant par femme en 2006-9 (INSTAT et al., 2010). La limitation des naissances s'est diffusée, mais le mariage est resté précoce, universel et étroitement lié à la constitution de la famille dans les années 1990 (Gjonca et al., 2008). Depuis la chute du système commu- 
niste, l'Albanie a en effet connu une retraditionalisation sociale. Celle-ci s'explique notamment par la volonté de défier le régime précédent en se réappropriant d'anciennes coutumes ainsi que par l'importance sociale de la réputation familiale jadis si déterminante sous le communisme (Nixon, 2009). Le rôle de la famille comme filet de sécurité sociale et économique a sans doute accentué cette retraditionalisation. Elle s'est manifestée durant les années 1990 par une anticipation temporaire des mariages - déjà précoces à l'époque (Lerch, 2013).

Durant les années de stabilisation économique et politique, les comportements reproductifs ont connu une profonde mutation. La fécondité albanaise a continué à chuter comme le témoigne le faible ISF dans les villes en 2006-9 : 1,0 enfant par femme à Tirana et 1,3 pour les autres villes du pays (INSTAT et al., 2010). Le mariage a progressivement été retardé (l'âge moyen a augmenté de 22 à 24 ans) et le modèle de la famille à enfant unique a commencé à se diffuser, particulièrement parmi les populations urbaines et plus éduquées. Une minorité de femmes continue toutefois à se marier très jeune et l'on observe une diversification croissante des comportements familiaux urbains (Lerch, 2013).

\section{Question de recherche et hypothèses}

L'exode rural provient des régions périphériques caractérisées par une fécondité élevée et concerne majoritairement des femmes (à hauteur de $60 \%$; INSTAT, 2004). Le changement des comportements féconds des migrantes lors de leur arrivée en ville est donc susceptible de soutenir la transition de la fécondité. Le but de cette analyse est de vérifier l'importance de cet effet comportemental lié à l'exode rural. Les effets de la migration sur les comportements reproductifs sont généralement conceptualisés dans une perspective de parcours de vie. Le mariage et la fécondité des migrantes sont susceptibles de diverger de ceux des non migrantes en raison de leurs expériences au cours des quatre étapes majeurs du parcours migratoire, à savoir : la socialisation préalable au lieu d'origine, la sélection migratoire, la rupture momentanée du parcours de vie due aux effets perturbateurs de la mobilité elle-même, ainsi que l'intégration au lieu d'accueil (Kulu, 2003 ; Goldstein, Goldstein, 1983). Au vu de l'évolution du contexte socioéconomique et démographique en Albanie, nous postulons des interactions différentes entre l'exode rural et la fécondité au cours des deux dernières décennies. 
Selon la théorie économique néoclassique, les migrants sont sélectionnés parmi les populations pour lesquelles les bénéfices escomptés dépassent les coûts du déplacement géographique (Sjaastad, 1962). Ils sont généralement plus éduqués et se caractérisent souvent par une fécondité inférieure à celle des non migrants au lieu d'origine. On évoque même l'hypothèse d'une sélection en fonction de la fécondité souhaitée. Les femmes, dont les souhaits de fécondité sont inférieurs à la moyenne au lieu d'origine mais semblable à celle généralement plus faible au lieu de destination urbaine, connaitraient des probabilités à l'émigration plus importante, tel qu'il l'a été observé au Pérou ou au Ghana (White et al., 1995 ; Chattopadhyay et al., 2006). Or, la constitution d'une famille peut aussi représenter la motivation même du projet migratoire, qui devient alors interdépendant avec l'évènement familial (Courgeau, 1985 ; Mulder, Wagner, 1993). Ainsi en Tchécoslovaquie communiste, les femmes immigrées dans les villes affichaient des niveaux de fécondité temporairement supérieurs aux non migrantes en raison de l'allocation des logements urbains aux jeunes familles (Boguszak et al., 1989). En l'absence d'aide institutionnelle, la migration des couples s'effectuent souvent par étape. L'établissement préalable de l'homme ou de la femme est suivi du mariage ou du regroupement familial ainsi que par un rattrapage du retard dans la réalisation des projets familiaux. De tels cas de figure sont avancés pour expliquer la fécondité plus élevée des migrantes à l'arrivée dans les villes du Guatemala et au Kirghizistan (Lindstrom, 2003 ; Nedoluzhko, Andersson, 2007).

Selon les hypothèses de sélection de femmes moins fécondes ou d'interdépendance entre évènements démographiques, les migrantes n'influencent guère la transition de la fécondité au niveau national. En effet, les femmes réalisent un projet fécond spécifique, prédéterminé selon leur profil socioéconomique et leurs valeurs au lieu d'origine. Les interdépendances entre comportements démographiques peuvent toutefois jouer un rôle sur la transition de la fécondité s'ils influencent les déterminants proches de celle-ci - tel l'âge au mariage. Ainsi, dans les années 1980 en Albanie, la surreprésentation féminine dans les villes a été attribuée aux migrations matrimoniales de femmes qui rejoignaient leur futur mari (Sjöberg, 1989). Ces traditions nuptiales exogames et patrilocales ont été instrumentalisées sous le communisme afin de contourner les restrictions de la mobilité interne (Sjöberg, 1994 ; Vullnerati, 2007). Au regard de la retraditionalisation de la société durant les années 1990, il est probable que cette interdépendance institutionnalisée entre migration et mariage ait perduré. Puisqu'elle intervient généralement à des âges jeunes et mène à une descendance nombreuse, les migrantes 
auraient maintenu une fécondité élevée et freiné ainsi la transition de la fécondité au niveau national.

Cependant, les comportements familiaux albanais se sont transformés parallèlement à l'amélioration de la situation économique et sociale durant la seconde décennie. Dans ce contexte de modernisation, les migrantes se sont peut-être mariées plus tardivement et l'émancipation par rapport aux normes culturelles est susceptible d'avoir baissé leur niveau de fécondité. Ainsi, l'importante chute de l'indice synthétique de fécondité dans les villes aurait été soutenue par un changement des comportements nuptiaux et des souhaits de fécondité chez les migrantes.

De même, la baisse de la fécondité des migrantes peut s'expliquer par des ruptures momentanées du parcours reproductif, en raison notamment de la séparation des époux et des conditions de vies difficiles en début de séjour au lieu d'accueil (Goldstein, Goldstein, 1983; Brockerhoff, 1998).

À plus long terme, le processus d'intégration structurelle et culturelle en ville joue un rôle déterminant. Plus la durée de résidence augmente, plus les comportements reproductifs des immigrants tendent à converger vers ceux de la population locale (Kulu, 2003). Les effets de l'intégration structurelle sont toutefois difficilement prévisibles en Albanie. Bien que bénéficiant d'un niveau de formation intermédiaire, les migrantes ont des difficultés d'insertion sur le marché du travail comparativement aux non-migrantes tant dans les zones d'origine que d'accueil. Nombreuses sont celles qui ont quitté le marché du travail durant la crise et se sont déclarées comme étant femme au foyer (INSTAT, 2004). La migration interne semble avoir transférer des populations pauvres des régions reculées vers les villes (Zezza et al., 2005). Bien que les migrants disposent d'un revenu supérieur aux non-migrants au lieu d'origine, leur niveau de consommation et leurs conditions de santé ou de logement ne se sont pas systématiquement améliorés (Hagen-Zanker, Azzarri, 2008). Ainsi, la fécondité pourrait soit augmenter en raison des faibles coûts d'opportunité, soit diminuer en raison des conditions de vie difficiles. Or, l'intégration culturelle des immigrantes est supposée baisser leur niveau de fécondité, puisque la vie urbaine s'est rapidement modernisée depuis l'ouverture internationale du pays en 1990. La transition de la fécondité s'en trouverait ainsi accélérée par le déplacement des populations des régions rurales vers les villes et par l'adaptation de leurs comportements reproductifs.

Toutefois, la fécondité des migrantes pourrait être moins déterminée par les nouvelles contraintes matérielles et sociales de la vie urbaine, y 
compris ses normes familiales, que par les valeurs auxquelles les migrantes ont été confrontées dans l'enfance. Puisque les régions d'origine sont souvent géographiquement reculées et sont restées isolées pendant des décennies, ces normes sociales feraient perdurer une fécondité élevée indépendamment du lieu de résidence ultérieur. En outre, les immigrants résident dans des zones urbaines spécifiques, comme à Tirana (Agorastakis, Sidiropoulos, 2007). Cette concentration spatiale des migrants a favorisé l'importation de leur culture rurale patriarcale afin de maintenir une identité collective et d'organiser la vie sociale dans des espaces souvent dépourvue d'infrastructures et de services urbains (Voell, 2003). Au vu de l'importance des ressources communautaires dans les récits d'adaptation des immigrants (Caro, 2011), les femmes pourraient ainsi rester soumises à une forte pression sociale promouvant le mariage précoce et la famille nombreuse. Les influences sociales du voisinage sur les comportements reproductifs jouent en effet un rôle non négligeable dans les villes en voie de développement (Montgomery et al., 2003). L'effet de socialisation serait ainsi soutenu au lieu de destination, ce qui retarderaient la transition de la fécondité au niveau national.

Nous postulons qu'un tel effet de socialisation a été dominant durant les années 1990, lorsque le processus d'urbanisation n'était qu'à ses débuts et la retraditionnalisation de la société plus marquée. L'émancipation des migrantes face à leur socialisation et l'adaptation de leurs comportements reproductifs devraient toutefois s'accroitre avec la transformation de l'habitat urbain, la modernisation de la société et la résidence prolongée des immigrantes en ville durant la seconde décennie. La fécondité des migrantes auraient ainsi convergé récemment vers les niveaux plus faibles des citadines de naissance. Ce phénomène devrait tout particulièrement concerner les enfants issus de l'immigration, tel que White et al. (2005) l'ont observé au Ghana, en raison de leur socialisation dans un contexte urbain davantage différencié.

\section{Données et méthodes}

Conformément à l'hypothèse de la transition de la mobilité, le rôle de l'exode rural dans la baisse de la fécondité albanaise est déterminé par une analyse transversale. Nous vérifions dans quelle mesure les comportements nuptiaux et féconds des femmes participant à l'exode rural diffèrent au cours de leur parcours migratoire des non migrantes dans les campagnes durant les deux dernières décennies. 
Nos hypothèses sont testées auprès de 5007 et 6690 biographies féminines récoltées respectivement durant l'enquête de santé et de reproduction en 2002 (Reproductive and Health Survey, RHS) et l'enquête démographique et de santé en 2008-9 (Demographic and Health Survey, DHS). Ces échantillons de femmes en âge reproductif sont représentatifs au niveau national ainsi que pour les zones urbaines et rurales. Ils ont été sélectionnés selon un échantillonnage aléatoire en grappe : une femme a été interviewée dans chacun des ménages tirés au hasard dans les aires d'énumération de recensement qui ont été sélectionnées proportionnellement au poids démographique des régions dans lesquelles elles se situent. Le taux de non-réponses est inférieur à $7 \%{ }^{3}$. Outre l'histoire génésique, les enquêtes fournissent des informations relatives à l'année (et au mois dans le RHS) de la dernière migration ainsi qu'au statut urbain du lieu d'origine et d'accueil ${ }^{4}$. Sont considérées comme migrantes, les femmes qui ont quitté une commune de résidence rurale depuis 1990. Étant donnée l'absence d'indications relatives au mois de la migration dans le DHS, on a considéré que l'évènement s'est produit au milieu de l'année. Les interrelations entre migration et fécondité sont testées auprès de deux cohortes synthétiques. La première de 1990 à 1999 est construite à partir du RHS et la seconde de 2000 à 2008 sur la base des données du DHS. Deux aspects de la reproduction sont étudiés : l'âge à l'entrée en vie féconde (soit le mariage) et l'intensité de la fécondité légitime.

Des modèles multivariés de survie en temps discret (mesuré en personnes-mois) sont spécifiés afin de tester les relations entre d'une part la migration et le mariage, et d'autre part la migration et une naissance de rang supérieur (seconde ou plus élevée; Allison, 1995). Nous estimons les rapports de côtes du mariage et des évènements génésiques. La durée d'exposition au risque est respectivement contrôlée par l'âge sous forme logarithmique et l'intervalle intergénésique sous forme linéaire et quadratique - chacune de ces variables évoluant dans le temps. L'exposition au risque d'un mariage débute après le 15ème anniversaire avec

3. Pour plus d'information voir Morris et al., 2005 et INSTAT et al., 2010.

4. Les femmes interviewées par le RHS ont répondu aux questions suivantes: «Depuis quand (année et mois) vivez-vous continuellement dans ce lieu de résidence ?» ; «Avant d'arriver ici, est-ce que vous avez vécu dans une ville, un village ou à l'extérieur de l'Albanie ?». Les six femmes venant de l'étranger ne sont pas considérées dans cette analyse. L'énoncé des questions du DHS ont été : «Depuis combien d'années vivez-vous continuellement dans ce lieu de résidence ?» ; «Avant d'arriver ici, est-ce que vous avez vécu dans une grande ville, une petite ville ou un village ?». Les femmes provenant d'une grande ou petite ville ont été regroupées dans la classe urbaine. Le statut urbain du lieu de destination équivaut à celui du lieu d'enquête. 
une troncature à 35 ans ; les intervalles intergénésiques sont tronqués après dix ans. L'exposition au risque est également tronquée à gauche par le début et à droite par la fin des périodes d'observation. Puisque le modèle de la fécondité analyse tous les intervalles intergénésiques ouverts par femme au cours des périodes d'observation, le modèle contrôle la parité atteinte qui évolue dans le temps, tout comme le statut et les étapes migratoires. En effet, tant les situations décisionnelles, que les projets féconds des femmes changent au cours du parcours reproductif et migratoire. L'âge n'est pas contrôlé dans le modèle de la fécondité car il est corrélé avec la parité et l'intervalle intergénésique durant les années 1990 ; en outre, l'introduction de cette variable n'affecte pas les rapports de côte selon le statut migratoire.

L'intérêt principal de cette analyse porte sur l'effet de la variable indexant l'émigration des zones rurales, ses étapes et ses générations. Les femmes non-migrantes dans les villes ou dans les campagnes sont distinguées de la première et de la 1,5ème génération d'émigrées des campagnes, qui se définissent par un départ respectivement aux âges de 15 ans ou plus depuis 1990 et de 6 à 14 ans depuis 1980. Divers effets de la migration sont testés à des étapes différentes du parcours migratoire. Les comportements familiaux au cours de la période qui précède d'au moins une année la migration indiquent d'éventuels reports des évènements en vue d'un déplacement géographique. La période migratoire est ensuite indexée par une fenêtre temporelle de deux ans centrés autour de la date de migration dans le modèle du mariage et de trois ans dans le modèle de la fécondité (soit respectivement une année précédant et deux années suivant le déplacement géographique). Si le risque d'un évènement familial est élevé durant cette période, il y a interrelation entre comportements migratoires et reproductifs (Mulder, Wagner, 1993) ; s'il est faible, on peut en déduire un effet de rupture ${ }^{5}$. La durée écoulée depuis la migration est désagrégée annuellement dans le modèle du mariage et triennalement dans le modèle de la fécondité. Les effets de l'adaptation structurelle et culturelle sont évalués en fonction du report du mariage et de la baisse de la fécondité associés à la durée de résidence. Dans le cas inverse, on peut conclure à un effet de socialisation. Enfin, les comportements de la 1,5ème génération de migrants

5. Des tests ont démontré que les effets de la migration sur le mariage et la fécondité sont similaires durant la période précédant ou suivant immédiatement le déplacement géographique. L'enchainement temporel des comportements migratoires et reproductifs peut en effet varier et les effets de rupture peuvent soit anticiper soit être consécutifs à une migration. 
confirment s'il y a eu adaptation aux normes urbaines ou si la socialisation est intergénérationnelle.

Puisque les données sont rétrospectives, la sélection des émigrantes selon leur projet familial ne peut pas être observée. Elle peut toutefois être testée par la comparaison du gradient rural-urbain estimé par deux modèles différents. Dans le premier, on distingue les non-migrantes en villes des femmes originaires de la campagne sans distinction du statut ni du parcours migratoire tel que défini précédemment. Le second modèle contrôle le statut d'émigration des campagnes. Si le déficit de fécondité urbaine par rapport aux non-migrantes des zones rurales est supérieur (inférieur) au déficit par rapport à l'ensemble des femmes originaires des campagnes, il y a sélection de femmes moins (plus) fécondes. L'importance de l'exode rural pour la compréhension des comportements familiaux en Albanie est évaluée en fonction de l'ajustement aux données du second modèle comparé au premier. Nous recourons au critère d'information Bayésien (BIC) qui est basé sur la statistique du rapport de vraisemblance et tient compte de la complexité du modèle et du nombre d'évènements étudiés. La destination de la migration n'est pas considérée, puisque la majorité des mouvements a contribué à l'exode rural (voir Tableau 1).

Les différentiels de mariage et de fécondité selon le statut migratoire sont contrôlés pour les effets confondant du niveau d'éducation achevé en distinguant le niveau post-obligatoire des niveaux inférieurs. La rupture du parcours génésique liée à une émigration internationale du conjoint est un biais potentiel en Albanie que nous ne sommes pas en mesure de contrôler, faute de données. Les résultats ne devraient toutefois pas être significativement affectés, car les migrants de longue durée sont majoritairement célibataires (et se marient probablement après leur retour), tandis que les hommes mariés partent davantage pour de courts séjours (Carletto et al., 2004). Puisque les principaux pays de destination sont frontaliers avec l'Albanie (l'Italie et la Grèce), les allers et retours sont fréquents et les naissances retardées ont le temps d'être récupérées.

$\mathrm{Au}$ vu de l'universalité du mariage en Albanie et de la baisse des naissances de rang supérieur, les rapports de côtes du mariage sont interprétés comme des différentiels de calendrier, tandis que les estimations relatives aux naissances de rang supérieur sont commentées en termes de différentiel d'intensité. 


\section{Résultats}

Les résultats des modèles de survie sont présentés dans le Tableau 2. Le rapport de côte du mariage augmente avec l'âge et la fécondité suit une courbe en $U$ inversée selon la durée écoulée depuis le dernier évènement génésique. La principale différence entre 1990-1999 et 2000-2008 concerne la fécondité de rangs supérieurs : après contrôle des effets des autres variables, le rapport de côte associée à la parité atteinte est inférieur dans la seconde décennie par rapport à la première, indiquant la diffusion de la limitation des naissances. Les femmes disposant d'un niveau de formation post-obligatoire se marient plus tardivement et ont une fécondité plus faible comparées aux femmes moins formées. Cette différentiation socioéconomique semble également se creuser dans le temps.

Les populations urbaines se marient plus tardivement que le groupe de référence constitué de la population d'origine rurale (modèle 1). Cela est particulièrement le cas en 1990-1999, tandis qu'en 2000-2008 la différence est faible et statistiquement non significative. Si la prise en compte du statut d'émigration de la population d'origine rurale augmente significativement l'ajustement du modèle pour la période 1990-1999 (comme l'indique la baisse du BIC ; Modèle 2), le gradient urbain-rural s'inverse étonnement parmi les non-migrantes: les femmes restées dans les campagnes se marient plus tardivement que celles des villes. Les émigrantes semblent donc être sélectionnées en fonction de l'âge au mariage. L'attente d'une émigration retarde le mariage jusqu'à l'année précédant le départ, mais ces deux évènements coïncident généralement parmi les migrantes et ceci à des âges plus jeunes comparés aux nonmigrantes. Les rapports de côtes du mariage sont si élevés durant la période migratoire qu'il est difficile de conclure à un effet de rattrapage, mais bel et bien à une interrelation entre comportements migratoires et nuptiaux. L'importante sélection migratoire selon l'intention de se marier jeune et l'interdépendance de cet évènement avec le départ en ville est susceptible d'expliquer l'étonnant retard du mariage parmi les femmes restées dans les campagnes. Elles attendent probablement, elles aussi, l'opportunité pour se marier et partir.

Lorsque ces deux évènements démographiques ne sont pas synchronisés, les migrantes se marient également plus jeunes au lieu de destination que les non migrantes - et cela quelle que soit la durée de séjour. L'effet de socialisation semble ainsi dominer les comportements nup- 
TABleau 2 Effets de l'émigration des femmes issues des zones rurales sur le mariage et la fécondité comparés au non migrantes selon le lieu de résidence, femmes 15-44 ans, Albanie 1990-1999 et 2000-2008

\begin{tabular}{|c|c|c|c|c|c|c|c|c|}
\hline & \multicolumn{8}{|c|}{ Mariage $^{a}$} \\
\hline & \multicolumn{4}{|c|}{ 1990-1999 } & \multicolumn{4}{|c|}{$2000-2008$} \\
\hline & \multicolumn{2}{|c|}{ Modèle 1} & \multicolumn{2}{|c|}{ Modèle 2} & \multicolumn{2}{|c|}{ Modèle 1} & \multicolumn{2}{|c|}{ Modèle 2} \\
\hline & $\mathrm{RC}$ & Sig & $\mathrm{RC}$ & Sig & $\mathrm{RC}$ & Sig & $\mathrm{RC}$ & Sig \\
\hline Âge (éch. log) & 26,61 & $* *$ & 14,91 & $* *$ & 11,39 & $* *$ & 7,27 & $* *$ \\
\hline \multicolumn{9}{|l|}{ Éducation } \\
\hline Au plus niv obl. & 1 & & 1 & & 1 & & 1 & \\
\hline Post-obligatoire & 0,75 & $* *$ & 0,76 & $* *$ & 0,55 & $* *$ & 0,57 & $* *$ \\
\hline \multicolumn{9}{|l|}{ Origine urbaine et statut migratoire } \\
\hline Urbain & 0,59 & $* *$ & 1,23 & $* *$ & 0,95 & ns & 3,01 & $* *$ \\
\hline Rural & \multicolumn{2}{|c|}{1} & & & \multicolumn{2}{|c|}{1} & & \\
\hline Non migrant & & & 1 & & & & 1 & \\
\hline 1,5ème génération d'émigrants & & & 1,48 & * & & & 1,43 & ns \\
\hline Jusqu'à 1 an avant l'émigration & & & 0,76 & $* *$ & & & 0,98 & ns \\
\hline Pendant l'émigration & & & 8,32 & $* *$ & & & 21,61 & $* *$ \\
\hline 2ème année après l'émigration & & & 2,33 & $* *$ & & & 32,54 & $* *$ \\
\hline $\begin{array}{l}\text { 3ème année ou plus après } \\
\text { l'émigration }\end{array}$ & & & 2,00 & $* *$ & & & 3,70 & $* *$ \\
\hline $\mathrm{N}$ tronqués & 168993 & & 168993 & & 163365 & & 163365 & \\
\hline$N$ évènements & 2014 & & 2014 & & 785 & & 785 & \\
\hline \multirow[t]{5}{*}{$\mathrm{BIC}$} & 21037 & & 19673 & & 9694 & & 8844 & \\
\hline & \multicolumn{8}{|c|}{ Naissances de rang supérieur ${ }^{b}$} \\
\hline & \multicolumn{4}{|c|}{ 1990-1999 } & \multicolumn{4}{|c|}{$2000-2008$} \\
\hline & \multicolumn{2}{|c|}{ Modèle 1} & \multicolumn{2}{|c|}{ Modèle 2} & \multicolumn{2}{|c|}{ Modèle 1} & \multicolumn{2}{|c|}{ Modèle 2} \\
\hline & $\mathrm{RC}$ & Sig & $\mathrm{RC}$ & Sig & $\mathrm{RC}$ & Sig & $\mathrm{RC}$ & Sig \\
\hline Parité atteinte & 0,90 & $* *$ & 0,89 & $* *$ & 0,49 & $* *$ & 0,51 & $* *$ \\
\hline $\begin{array}{l}\text { Année depuis dernier évènement } \\
\text { génésique }\end{array}$ & 1,09 & $* *$ & 1,10 & $* *$ & 1,07 & $* *$ & 1,07 & $* *$ \\
\hline Au carré & 0,999 & $* *$ & 0,999 & $* *$ & 0,999 & $* *$ & 0,999 & $* *$ \\
\hline \multicolumn{9}{|l|}{ Éducation } \\
\hline Au plus niv obl. & 1 & & 1 & & 1 & & 1 & \\
\hline Post-obligatoire & 0,85 & $* *$ & 0,85 & $* *$ & 0,61 & $* *$ & 0,60 & $* *$ \\
\hline \multicolumn{9}{|l|}{ Origine urbaine et statut migratoire } \\
\hline Urbain & 0,81 & $* *$ & 0,82 & $* *$ & 0,63 & $* *$ & 0,76 & $* *$ \\
\hline Rural & 1 & & & & 1 & & & \\
\hline Non migrant & & & 1 & & & & 1 & \\
\hline 1,5ème génération d'émigrants & & & 0,84 & ns & & & 2,28 & $* *$ \\
\hline Jusqu'à 1 an avant l'émigration & & & 1,10 & ns & & & 0,65 & $*$ \\
\hline Pendant l'émigration & & & 1,02 & ns & & & 0,94 & ns \\
\hline 3-5 années après l'émigration & & & 0,98 & ns & & & 1,37 & $* *$ \\
\hline 6-8 années après l'émigration & & & 0,97 & ns & & & 1,34 & $* *$ \\
\hline 9 années ou plus après l'émigration & & & & & & & 1,14 & ns \\
\hline $\mathrm{N}$ tronqués & 93233 & & 93233 & & 269287 & & 269287 & \\
\hline $\mathrm{N}$ évènements & 2188 & & 2188 & & 1487 & & 1487 & \\
\hline $\mathrm{BIC}$ & 19717 & & 19752 & & 17367 & & 17375 & \\
\hline
\end{tabular}

Source : RHS 2000 \& DHS 2008/9.

Notes : Les individus ayant migré à l'âge de 15 ans ou plus avant 1990 sont exclus; RC: Rapport de côte ; ${ }^{+}$: N'évolue pas dans le temps; ${ }^{*}$ ou ${ }^{* *}$ : Statistiquement significatif à $90 \%$ ou $95 \% ;{ }^{a}: 15-34$ ans $;{ }^{\text {b }}$ : Les intervalles sont tronqués après neuf années écoulées depuis l'évènement génésique précédant. 
tiaux des femmes participant à l'exode rural. Puisque les enfants des émigrants, partiellement socialisés en ville, se marient également plus jeunes, on peut conclure à un effet de socialisation intergénérationnelle.

Contrairement à notre hypothèse, la sélection migratoire et son interdépendance avec un mariage précoce semble se renforcer au lieu de s'atténuer durant la période 2000-2008 : les migrantes se marient d'autant plus jeunes par rapport aux non migrantes. Les effets de socialisation persistent également. Après l'émigration, le rapport de côte du mariage diminue certes avec la durée de résidence, mais reste très élevé par rapport aux femmes restées dans les campagnes. Cependant, l'anticipation du mariage parmi les enfants issus de l'émigration n'est statistiquement plus significative en 2000-2008, indiquant une tendance vers l'adaptation des comportements aux normes urbaines.

Comme pour le mariage, les non-migrantes des villes ont un niveau de fécondité inférieur à celui des femmes originaires des campagnes. Le contrôle du statut migratoire n'améliore pas l'ajustement du modèle. On n'observe d'ailleurs aucune différence significative de fécondité selon le statut d'émigration, la durée de résidence ou la génération de migrants en 1990-1999. Cela confirme un effet de socialisation. Durant la seconde décennie, le différentiel urbain-rural se creuse, mais se réduit légèrement après contrôle du statut migratoire. Les émigrantes semblent donc être davantage sélectionnées dans les régions rurales parmi les femmes désirant plus d'enfants en 2000-2008. En effet, les différentiels de fécondité selon le statut d'émigration sont significatifs. Si les candidates à l'exode rural reportent les naissances en vue d'un départ, celles-ci sont récupérées durant la période d'intégration structurelle et particulièrement durant les années consécutives. Le niveau de fécondité des migrantes est significativement supérieur à celui des non-migrantes entre la troisième et la neuvième année de résidence, ce qui indique un effet dominant de la socialisation aux normes familiales rurales ${ }^{6}$. La fécondité élevée des enfants issus de l'émigration des campagnes confirme cette interprétation, bien qu'il s'agisse ici en partie d'un effet générationnel. Certaines de ces femmes aux parités supérieures se sont mariées durant la décennie précédente et continuent à afficher des comportements familiaux traditionnels.

6. Puisque l'approche est transversale, le différentiel de fécondité associé à la durée de séjour confond les expériences de deux types de migrantes différentes : celles qui ont migré à l'occasion de leur mariage et celle qui ont quitté les campagnes ultérieurement aux parités supérieures. Des analyses séparées pour les deux groupes ont démontré le même effet de socialisation sur leur fécondité (non montré). 


\section{Discussion et conclusion}

Parallèlement à la réémergence des mouvements migratoires depuis la fin du régime communisme en Albanie, la fécondité a diminué de moitié. Des populations plus fécondes se sont déplacées des régions périphériques vers les villes et leurs alentours, où elles ont été confrontées à de nouveaux styles de vie et à de nouvelles contraintes matérielles ou sociales susceptibles de motiver le désir d'une famille plus restreinte. Notre analyse a tenté de vérifier dans quelle mesure l'exode rural a contribué à la baisse de la fécondité albanaise au niveau national. Le calendrier du mariage et l'intensité de la fécondité des non-migrantes ont été comparés à ceux des migrantes issues des campagnes en considérant leur parcours migratoire ainsi que la génération de migrants. Nous avons postulé que les interactions entre migration et fécondité ont évolué dans le temps au gré des transformations de l'Albanie depuis 1989. Durant la première décennie, marquée par des crises économiques et politiques, nous avons émis l'hypothèse d'une interdépendance entre l'émigration rurale et le mariage traditionnel ainsi qu'une fécondité élevée parmi les migrantes en raison d'un effet de socialisation dominant. Durant les années consécutives de stabilisation économique et de modernisation sociale, nous avons supposé une émancipation des migrantes par rapport aux normes traditionnelles et une adaptation accrue de leurs comportements aux réalités urbaines. Or, nos résultats ne montrent guère de telles évolutions entre les deux périodes.

L'exode rural des femmes a été très sélectif et cela en fonction des projets familiaux. Il a non seulement concerné celles désirant se marier à un jeune âge et avoir une famille nombreuse, mais leur mariage a aussi coïncidé avec le départ des campagnes. Au lieu de diminuer en importance, la sélection démographique et la synchronisation des évènements ont été maintenues comme dans le passé socialiste, si ce n'est de manière plus marquée encore. Ces résultats contrastent avec la diffusion du retard au mariage observée dans le pays. Ainsi, le changement historique des comportements nuptiaux semble concerner uniquement les non-migrantes. Le contexte urbain n'a pas non plus eu d'impact significatif sur la fécondité légitime des migrantes. C'est la socialisation aux comportements reproductifs dans les régions d'origine qui a déterminé leur niveau de fécondité au lieu de destination. Comme pour le mariage, le différentiel de fécondité selon le statut migratoire s'accroît lorsque le modèle familial à enfant unique se diffuse durant la seconde décennie. Aucun effet de rupture et d'adaptation au contexte urbain n'est observé. Ces phénomènes sont non seulement soutenus dans le temps mais aussi 
à travers les générations de migrantes. On décèle toutefois une tendance vers la convergence amorcée par la dernière cohorte d'enfants issus de l'émigration et socialisée dans un contexte davantage urbain.

L'étroite interdépendance entre migration et mariage précoce, ainsi que la sélection migratoire en fonction des projets familiaux, soulignent l'importance des migrations traditionnelles alors même que ré-émergent en Albanie des formes modernes de mobilité, tel que l'exode rural et la migration internationale de la main-d'œuvre. Si ce constat concurrence les effets supposés de la modernisation et les théories économiques de la mobilité, il s'inscrit dans un contexte spécifique. L'Albanie s'est caractérisée par une soudaine ouverture internationale, un rattrapage de l'urbanisation auparavant freinée par le régime communiste ainsi que par des traditions nuptiales exogames et patrilocales. Le pays a connu d'importantes transformations socioéconomiques dans un contexte de fortes pressions démographiques durant la crise institutionnelle et économique qui ont suivi l'effondrement du communisme. Suite à la levée des restrictions administratives de la mobilité, de futures conjointes ou jeunes couples mariés ont quitté les régions rurales soudainement dépourvues d'emplois et de services jadis administrés sous la planification centrale. L'extraversion internationale du pays a renforcé la position privilégiée des villes comme moteur de développement économique et de changement social, attirant les jeunes adultes des campagnes pour réaliser leur projet familiaux dans un environnement nouveau.

Le nouvel essor des migrations matrimoniales s'insère également dans un contexte social marqué d'une réaffirmation de la culture patriarcale albanaise. Le mariage traditionnel a peut-être constitué un prérequis social au départ de jeunes femmes du foyer parental. Lors de l'arrivée dans les villes, les migrantes ont soit maintenu leur préférence de fécondité, soit sont restées sous l'influence sociale d'une communauté d'immigrés faisant perdurer ainsi des normes et des traditions rurales dans le nouvel espace de vie urbain. En outre, le faible coût d'opportunités des enfants pour les migrantes est susceptible de soutenir ces effets culturels et sociaux sur leur fécondité.

Une autre explication stipulerait une interrelation spécifique entre migration internationale, exode rural et fécondité. En effet, l'émigration internationale est dominée par les hommes tandis que les mouvements internes concernent principalement les femmes (INSTAT 2004). Moyennant l'hypothèse d'une orientation traditionnelle des émigrés internationaux, les comportements reproductifs des migrantes internes pourraient avoir résultés de mariages transnationaux entre un émigré et une Albanaise au pays d'origine, suivit du regroupement familial et de la réa- 
lisation des projets féconds au lieu de retour des émigrés. En effet, ceuxci préfèrent s'installer dans les zones économiquement centrales que de retourner dans leurs régions reculées d'origine, entraînant ainsi une migration interne de l'épouse vers les centres urbains.

Quelques soient les raisons de la résistance des femmes participant à l'exode rural face aux transformations que connait la famille albanaise contemporaine, cette analyse a montré que le contexte socioéconomique et culturel peut jouer un rôle non négligeable sur les interactions entre migration et fécondité. Alors que l'urbanisation et la baisse de la fécondité en Albanie semblaient associées au niveau macro-démographique, la transition de la fécondité est principalement attribuable à la transformation des comportements familiaux de femmes non-migrantes tant dans les campagnes que dans les villes. L'exode rural a freiné la transition de la fécondité au lieu de la soutenir. Elle n'a pas altéré les comportements familiaux des migrantes, mais est resté liée à la constitution d'une famille albanaise traditionnelle menant à une descendance nombreuse. Ainsi, l'association entre migration et mariage semble avoir affecté la transition de la fécondité par un effet à long terme à travers l'entrée précoce en vie reproductive. Dans une société patriarcale, la constitution d'une famille à un jeune âge peut avoir des répercussions sur l'ensemble du parcours fécond, comme l'indique les effets de socialisation persistants sur la fécondité des migrantes en ville. Tant la sélection migratoire que son interdépendance avec le mariage patriarcal pourraient être à l'origine de ces effets de socialisation. Nos résultats soulignent donc une étroite interrelation entre les différents effets de la migration sur la fécondité qui mériterait davantage d'investigation.

\section{Références}

Agorastakis M., Sidiropoulos G. (2007), «Population Change due to Geographic Mobility in Albania, 1989-2001, and the Repercussion of Internal Migration for the Enlargement of Tirana», Population, Space and Place, 13, pp. 417-418.

Aluson P. D. (1995), Survival Analysis Using the SAS System: A Practical Guide, Cary, NC, SAS Institute Inc.

Boguszak M., Courgeau D., Lelièvre E. (1989), «Constitution de la famille et urbanisation en Tchecoslovaquie: comparaison avec la France», Population, 44 (2), pp. 331-333. 
Brockerhoff M. (1998), "Migration and the Fertility Transition in African Cities», Migration, Urbanization, and Development: New Directions and Issues, R. E. BILSBORROW (ed), Norwell, KluwerAcademic Publishers, Massachusetts.

Calogero C., Davis B., Stampini M. et al. (2004), «Internal Mobility and International Migration in Albania», ESA Working Paper No. 04-13, The Food and Agriculture Organization of the United Nations.

Caro E. (2011), From the Village to the City: Adjustment Process of Internal Migrants in Albania, Rijksuniversiteit Groningen.

Central Directory of Statistics (1991), Population and Housing Census 1989: Principal Results, Republic of Albania, Tirana.

Chattopadhyay A., White M. J., DebpuUR C. (2006), "Migrant Fertility in Ghana: Selection Versus Adaptation and Disruption as Causal Mechanisms», Population Studies, 60 (2), pp. 189-203.

Courgeau D. (1985), «Interaction between Spatial Mobility, Family and Career LifeCycle: A French Survey», European Sociological Review, 1 (2), pp. 139-162.

Falkingham J., Guonca A. (2001), "Fertility Transition in Communist Albania, 1950$90 »$, Population Studies, 55 (3), pp. 309-318.

Gjonca A., Aassve A., Mencarinı L. (2008), «Albania: Trends and Patterns, Proximate Determinants and Policies of Fertility Change», Demographic Research, 91 (11), pp. 261-292.

Goldstein S., Goldstein A. (1981), «The Impact of Migration on Fertility: An «Own Children» Analysis for Thailand», Population Studies, 35 (2), pp. 265-284.

Goldstein S., Goldstein A. (1983), «Migration and Fertility in Peninsular Malaysia: An Analysis Using Life History Data», A Rand Note, Santa Monica (CA), Rand.

HAgen-Zanker J., AzzarRi C. (2008), «Are Internal Migrants in Albania Leaving for the Better?», Working paper, Maastricht University, Maastricht.

INSTAT (2004), Migration in Albania, Tirana, Institute of Statistics Albania.

INSTAT, PHI, ICF MACRO (2010), Albania Demographic and Health Survey 2008-09, Institute of Statistics (INSTAT), Institute of Public Health (PHI) and ICF Macro, Tirana.

INSTAT, UNDP, WoRLd BANK (2009), Albania, Trends in Poverty 2002-2005-2008, Tirana, INSTAT.

KULU H. (2003), "Migration and Fertility: Competing Hypotheses Re-examined», MPIDR Working Paper, 2003-035, 40 p.

LERCH M. (2013), «Fertility Decline During Albania's Societal Crisis and its Subsequent Consolidation», European Journal of Population, Online-first.

LINDSTROM D. P. (2003), «Rural-Urban Migration and Reproductive Behavior in Guatemala», Population Research and Policy Review, 22, pp. 351-372. 
Montgomery M. R., Stren R., Cohen B., Reed H. E. (2003), Cities Transformed: Demographic Change and Its Implications in the Developing World, Panel on Urban Population Dynamics, The National Academies Press, Washington D. C.

Morris L., Herold J., Bino S., Yili A., Jackson D. (2005), Reproductive Health Survey Albania 2020 - Final Report, Institute of Public Health (IPH), Albania Ministry of Health, Institute of Statistics (INSTAT), Tirana.

Mulder C., WAgner M. (1993), "Migration and Marriage in the Life Course: A Method for Studying Synchronized Events», European Journal of Population, 9 (1), pp. 55-76.

Nedoluzhro L., Andersson G. (2007), «Migration and First-Time Parenthood: Evidence from Kyrgyzstan», Demographic Research, 17 (25), pp. 741-774.

Nixon N. (2009), « «You Can't Eat Shame with Bread»: Gender and Collective Shame in Albanian Society», Southeast European and Black Sea Studies, 9 (1-2), pp. 105121.

SARDon J.-P. (2000), "L'évolution démographique des Balkans depuis la fin de la décennie 1980», Population, 55 (4-5), pp. 765-786.

Schuler M., Jarne A., Shameti E., Seferkoll L. (2010), «Typology of Communes and Definition of Agglomerations in Albania», Socio-Demographic Statistics in Albania: Selected Topics and Future Developments, INSTAT, Shtëpia Botuese \& Shtypshkronja, Tirana.

SJAASTAD L. A. (1962), "The Costs and Returns of Human Migration», The Journal of Political Economy, 70 (5), pp. 80-93.

Suöberg O. (1989), «A Note on the Regional Dimension of Post-War Demographic Development in Albania», Nordic Journal of Soviet \& East Europen Studies, 6 (1), pp. 91-121.

SJÖBERG O. (1992), «Underurbanisation and the Zero Urban Growth Hypothesis: Diverted Migration in Albania», Geografiska Annaler. Series B, Human Geography, 74 (1), pp. 3-19.

SJÖBERG O. (1994), «Rural Retention in Albania: Administrative Restrictions on Urban-Bound Migration», East European Quaterly, 28 (2), pp. 205-233.

UNDP (2000), Albanian Human Development Report, Tirana, UNDP.

Voell S. (2003), "The Kanun in the City. Albanian Customary Law as a Habitus and Its Persistence in the Suburb of Tirana, Bathore», Anthropos, 98 (1), pp. 85-101.

VullneratI J. (2007), "Albanian Migration and Development: State of the Art Review», Working Paper, IMISCOE.

White M. J., MoReno L., Guo S. (1995), «The Interrelation of Fertility and Geographic Mobility in Peru: A Hazards Model Analysis», International Migration Review, 29 (2), pp. 492-514. 
White M. J., Tagoe E., Stiff C., Adazu K., Smith D. J. (2005), «Urbanization and the Fertility Transition in Ghana», Population Research and Policy Review, 24, pp. 5983.

World Bank (2003), Albania Poverty Assessment, Washington D. C., World Bank.

WorLD BANK (2007), Albania: Urban Growth, Migration and Poverty Reduction. Washington: Poverty Reduction and Economic Management Unit, Europe and Central Asia Region, World Bank, New York.

Zezza A., Carletto G., Davis B. (2005), «Moving Away from Poverty: A Spatial Analysis of Poverty and Migration in Albania", Journal of Southern Europe and the Balkans, 7 (2), pp.175-193. 